\title{
Circulating miR-103a-3p and miR-660-5p are associated with bone parameters in patients with controlled acromegaly
}

\author{
Elena Valassi ${ }^{1}$, Natalia García-Giralt ${ }^{2}$, Jorge Malouf ${ }^{3}$, Iris Crespo ${ }^{1}$, Jaume Llauger ${ }^{4}$, Adolfo Díez-Pérez² and \\ Susan M Webb' \\ ${ }^{1}$ Endocrinology/Medicine Department, Hospital Sant Pau, Centro de Investigación Biomédica en Red de Enfermedades Raras (CIBERER, Unidad 747), \\ IIB-Sant Pau, ISCIII and Universitat Autònoma de Barcelona (UAB), Barcelona, Spain \\ 2URFOA, IMIM (Institut Hospital del Mar d'Investigacions Mèdiques), Universitat Autònoma de Barcelona, Barcelona, Spain \\ ${ }^{3}$ Mineral Metabolism Unit, Medicine Department, Hospital Sant Pau, Barcelona, Spain \\ ${ }^{4}$ Radiology Department, Hospital Sant Pau, Barcelona, Spain
}

Correspondence should be addressed to E Valassi: evalassi@santpau.cat

\begin{abstract}
Background: Biochemical control of GH/IGF-I excess in acromegaly (ACRO) is associated with persistent impairment of trabecular microstructure leading to increased risk of vertebral fractures. Circulating miRNAs modulate the activity of osteoblasts and osteoclasts, and may be potential biomarkers of osteoporosis.

Aims: Identify differentially expressed miRNAs in the serum of patients with controlled ACRO vs controls and correlate miRNA levels with both biochemical and structural bone parameters.

Patients and methods: Twenty-seven patients with controlled ACRO (11 males, 16 females; mean age, $48 \pm 5$ years; BMI, $28 \pm 4 \mathrm{~kg} / \mathrm{m}^{2}$ ) and 27 age-, gender- and BMI-matched controls were recruited. Areal BMD at lumbar spine and femur, and trabecular bone score were assessed; volumetric BMD was measured by quantitative computed tomography QCT-Pro (Mindways). Twenty miRNAs, chosen by their putative role in bone, were quantified in serum using real-time qPCR.

Results: In ACRO patients, miR-103a-3p and miR-191-5p were found overexpressed, whereas miR-660-5p was underexpressed $(P<0.001)$. miR-103a-3p levels were negatively associated with both trabecular VBMD at trochanter and serum osteoprotegerin concentrations $(P<0.05)$ and positively with vitamin D concentrations $(P<0.01)$ and total cross-sectional area of the femoral neck $(P<0.05)$. miR-660-5p levels were correlated with both trabecular vBMD at trochanter and OPG concentrations $(P<0.05)$, but were negatively associated with vitamin $D$ levels $(P<0.05)$. A negative correlation between miR-103-a-3p and miR-660-5p was found in both groups $(P<0.001)$.

Conclusions: Circulating miR-103a-3p and miR-660-5p are differentially expressed in controlled ACRO patients and associated with bone structural parameters. miRNAs may be one of the mechanisms involved in the pathogenesis of bone disease and could be used as biomarkers in ACRO patients.
\end{abstract}

\section{Key Words}

- acromegaly

- microRNAs

- volumetric bone mineral density 


\section{Introduction}

Growth hormone $(\mathrm{GH})$ and insulin-like growth factor-I (IGF1) are anabolic hormones which influence bone metabolism through the modulation of several signaling pathways (1). In vitro studies showed that GH enhances the proliferation and differentiation of osteoblastic cell lines driving the commitment of the mesenchymal stem cell (MSC) to the osteoblastogenesis $(1,2,3)$. On the other hand, IGF1 enhances the function of mature osteoblasts and maintains appropriate levels of bone matrix and bone mass (1).

Acromegaly (ACRO) is a rare disease caused by excessive GH production from a pituitary adenoma (4). Chronic elevation of GH and IGF1 in ACRO is associated with severe cardiovascular, respiratory and metabolic complications, which lead to an average 10-year reduction of life expectancy and a double standardized mortality rate as compared with the general population (5). Bone abnormalities are also a frequent manifestation of ACRO due to the deleterious effects of $\mathrm{GH}$ excess on bone remodeling and calcium homeostasis (1).

ACRO patients show increased bone turnover and deterioration of both biomechanical competence and microarchitecture at the trabecular compartment $(3,6,7)$. Of note, the latter has been associated with an increased prevalence of vertebral fractures even in the controlled patients, in the presence of normal lumbar areal bone mineral density (aBMD) (7). Cortical bone also appears to be impaired, in that a decrease in both bone material strength index (a marker of cortical bone properties), as assessed using microindentation, and femoral cortical volumetric bone mineral density (vBMD) have been described in ACRO patients despite biochemical control of the disease $(8,9)$.

miRNAs are single-strand, non-coding RNAs that span between 19 and 24 nucleotide bases (10). These molecules are involved in the modulation of a wide variety of biological processes, including bone cell formation, bone remodeling, bone homeostasis and skeletal development (10). In particular, subsets of miRNAs influence the commitment and differentiation of the MSC into the osteogenic lineage by regulating several molecular pathways (11). Indeed, the deregulation of miRNA-mediated mechanisms has been described as an important pathogenic factor for bone degeneration and skeletal disease (11). Interestingly, miRNAs also circulate in serum as extracellular nuclease-resistant entities through a variety of carriers, such as exosomes, apoptotic bodies and vesicle-free lipoprotein (12).
As a result, miRNAs have been advocated as promising biomarkers to predict onset and progression of chronic disease, including osteoporosis (13). Different levels of circulating miRNAs ('signatures') have been found in patients with both idiopathic (10) and postmenopausal osteoporosis $(10,14,15,16)$ and have been indicated as reliable predictors of fragility fractures in postmenopausal women with and without type 2 diabetes (10, 17, $18,19,20,21)$. Belaya et al. recently analyzed bone samples from patients with active ACRO and found significant changes in the expression of several miRNAs involved in osteoblastogenesis (22). However, whether some circulating miRNAs are associated with bone abnormalities in ACRO and, therefore, could represent useful markers to predict persistent bone impairment in these patients is still to be determined.

Thus, our cross-sectional study was aimed at evaluating if there are differentially expressed miRNAs in the serum of patients with controlled ACRO as compared with sex-, age- and BMI-matched healthy controls. In addition, we examined if these differentially expressed miRNAs are related to bone parameters in our patients, including bone turnover markers, vBMD at spine and femur and trabecular bone score (TBS), a tool designed to evaluate trabecular bone quality from the lumbar spine DXA image.

\section{Subjects and methods}

\section{Subjects}

We studied 27 patients (16 females and 11 males) with controlled ACRO who have been recruited successively while attending our clinic. Eighteen of these patients (66\%) had been evaluated in previous studies from our center $(8,23)$.

Controlled ACRO was defined as IGF1 concentrations within the specific age-adjusted reference range, and in those patients who were not on GH receptor antagonist, random GH concentrations were lower than $1 \mathrm{ng} / \mathrm{mL}$. When the $75 \mathrm{~g}$ oral glucose load test was performed, the $\mathrm{GH}$ value equal to or $<0.4 \mathrm{ng} / \mathrm{mL}$ were considered suggestive of cured disease (24). All had a GH-secreting pituitary tumor confirmed pathologically.

Five patients were on pharmacological treatment: three on somatostatin analogs (SSa) and the other two were on combination therapy with a SSa and either cabergoline or pegvisomant. All patients had transsphenoidal surgery from 6 months to 25 years (median: 5 years) prior to the

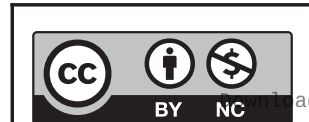


inclusion in the study, and seven of them also received radiotherapy from 7 to 24 years (median: 16 years) after unsuccessful surgery. Three patients had secondary adrenal insufficiency, and all of them were on stable replacement dose of hydrocortisone. Five patients had secondary hypothyroidism and were on stable replacement therapy with levothyroxine at the time of the study. Four males had secondary hypogonadism. Because all of them were on stable testosterone replacement for more than 1 year, they were considered eugonadal. Nine women had regular menses and seven were postmenopausal. None of the patients had GH deficiency, diagnosed when IGF-I levels were below two standard deviations for the agesex normal range in a patient with at least three other documented hormone deficiencies or with a peak plasma $\mathrm{GH}$ less than $3 \mu \mathrm{g} / \mathrm{L}$ during and insulin tolerance test (ITT) (25).

Mean ( \pm S.D.) duration of disease was $164 \pm 119$ months and was estimated as the time elapsed between the onset of symptoms and signs of ACRO (evaluated through old photographs and clinical history) and the time when treatment was proven to be effective. Mean ( \pm s.D.) duration of control was $146 \pm 121$ months and was calculated from the time between hormone normalization and study entry.

Twenty-seven age-, gender- and BMI-matched controls were also studied, recruited through advertisements at the blood donor center of our Hospital.

Control subjects who reported fragility fractures or who had morphometric vertebral fractures of any grade were excluded from the study. A fragility fracture was defined as any low-energy fracture, excluding those of the hands, feet and skull.

Patients and controls with malignancies, inflammatory disorders, kidney or liver dysfunctions were also excluded from the study.

Consent has been obtained from each patient or subject after full explanation of the purpose and nature of all procedures used.

This study was approved by the Ethical Committee of our institution (CEIC-IIB SantPau).

\section{Biochemical measurements}

Serum IGF1 concentrations were measured by an enzyme immunoassay (Mediagnost, Reutlingen/Germany) with a sensitivity of $0.09 \mathrm{ng} / \mathrm{mL}$. The intra- and interassay coefficients of variation (CVs) were 6.7 and 6.8\%, respectively. In the study, IGF-I is expressed as SD score (SDS).
Growth hormone (GH), osteocalcin, carboxyterminal collagen crosslinks (CTx) and total procollagen type 1 amino-terminal propeptide (P1NP) were measured by electrochemiluminescent immunoassay (cobas e601; Roche Diagnostics $\mathrm{GmbHm}$ ). Imprecision for mean GH values between 0.18 and $35 \mu \mathrm{g} / \mathrm{L}$ was $3-3.4 \%$, for osteocalcin concentrations between 6.11 and $160 \mu \mathrm{g} / \mathrm{L}$ was $2-2.3 \%$, for $\beta$-crosslaps concentrations between 0.06 and $4.64 \mu \mathrm{g} / \mathrm{L}$ was $5.7-2.4 \%$ and for total P1NP values between 14.4 and $1090 \mu \mathrm{g} / \mathrm{L}$ was $3.7-3.4 \%$. Sensitivity was $0.05 \mu \mathrm{g} / \mathrm{L}$ for $\mathrm{GH}, 0.5 \mu \mathrm{g} / \mathrm{L}$ for osteocalcin, $0.01 \mu \mathrm{g} / \mathrm{L}$ for CTx and $5 \mu \mathrm{g} / \mathrm{L}$ for P1NP. Serum 25-hydroxyvitamin D (vitamin D) concentrations were determined using an enzyme immunoassay (IDS, Boldon, UK), with a sensitivity of $4.8 \mathrm{ng} / \mathrm{mL}$. Imprecision for mean vitamin D values between 12 and $77.9 \mathrm{ng} / \mathrm{mL}$ was $9.9-7.7 \%$.

Serum PTH concentrations were measured by electrochemiluminescent immunoassay (cobas e601; Roche Diagnostics $\mathrm{GmbHm}$ ). Imprecision for mean PTH values between 23.2 and $184 \mathrm{pg} / \mathrm{mL}$ was $3.4-1.7 \%$. Serum osteoprotegerin (OPG) concentrations were measured by an enzyme-linked immune-sorbent assay (Abcam), with a sensitivity $<1 \mathrm{pg} / \mathrm{mL}$ and range between 1.23 and $900 \mathrm{pg} / \mathrm{mL}$.

Both serum Dickkopf-related protein 1 (DKK1) and sclerostin (SOST) concentrations were measured using an enzyme immunoassay (Biomedica, Vienna, Austria). Both intra- and inter-assay CVs for DKK1 were 3\% and the sensitivity was $1.7 \mathrm{pmol} / \mathrm{L}$. Intra- and interassay CVs for SOST were $<7$ and $<10 \%$, respectively; sensitivity was $3.2 \mathrm{pmol} / \mathrm{L}$. Serum osteoprotegerin concentrations were measured using an enzymelinked immune-sorbent assay (Biomedica). Intra- and interassay CVs were $<3$ and $<5 \%$, respectively; sensitivity was $0.07 \mathrm{pmol} / \mathrm{L}$.

\section{Radiological imaging}

Areal BMD (aBMD) was measured by dual-energy X-ray absorptiometry scanning (Hologic Discovery DXA system, HOLOGIC, Bedford, MA, USA). The CV was $1 \%$. Patients had the lumbar spine and femoral neck scanned. The scan acquisition and analysis were performed by a certified and experimented technician and were performed according to the ISCD standards. (http://www. iscd.org/documents/2015/06/2015-iscd-adult-officialpositions.pdf).

Trabecular bone score was acquired from the lumbar spine DXA image automatically by the TBS iNsight software, Medimaps Group SA, Geneva, Switzerland. 
Quantitative computed tomography (QCT) was performed using a Phillips Brilliance 16 scanner. Participants were positioned supine on the scanner table, lying on top of a solid calibration phantom which covered levels L2-L4 (Mindways Software, Inc. Austin, TX, USA). Spine volumetric BMD was calculated as the mean of the values at L2, L3 and L4 (expressed as LS vBMD). To measure femoral vBMD, all the scans were acquired from the acetabulum directly above the femoral head down to $1 \mathrm{~cm}$ below the lesser trochanter, resulting in 25-35 slices, with $3 \mathrm{~mm}$ slice thickness, over a range of $8-12 \mathrm{~cm}$. All scans were performed at $120 \mathrm{kV}$ and 70 to $200 \mathrm{mAs}$ depending on height and weight of the patient, according to the Mindways technical specifications. The images were processed and analyzed using QCT-pro Software version 4.1.3 and the QCT-pro Bone Investigational Toolkit Version 2.0 (BIT, Mindways Software, Inc.) by the same physician (JM). Volumetric BMD was obtained from the hip QCT analysis performing the following automated steps: (a) extraction of the proximal femur and (b) rotation and segmentation of bone voxels from soft tissue in three planes (axial, sagittal and coronal). For each scan at each time point, a fixed threshold $\left(450 \mathrm{mg} / \mathrm{cm}^{3}\right)$ was used to discriminate cortical from trabecular compartment (26). Mechanical properties were obtained using the BIT software. A sliced narrow neck (NN) analysis was performed and the mechanical properties (buckling ratio (BR), cross-sectional area $\left(\mathrm{CSA} ; \mathrm{cm}^{2}\right)$ and average cortical thickness (ACT, cm)) were the mean value of the NN series results. NN consists of nine slices of the femoral neck; the average of the slice structural results was used to perform the analysis.

\section{miRNA isolation from serum samples}

RNA extraction was conducted at Exiqon Services, Denmark. Serum samples were centrifuged at $3000 \boldsymbol{g}$ for $5 \mathrm{~min}$ at $4^{\circ} \mathrm{C}$. An aliquot of $200 \mu \mathrm{L}$ per sample was transferred to a FluidX tube and $60 \mu \mathrm{L}$ of Lysis solution BF containing $1 \mu \mathrm{g}$ carrier-RNA per $60 \mu \mathrm{L}$ Lysis Solution BF and RNA spike-in template mixture was added to the sample and mixed for $1 \mathrm{~min}$ and incubated for $7 \mathrm{~min}$ at room temperature, followed by addition of $20 \mu \mathrm{L}$ Protein Precipitation solution BF. Total RNA was extracted from the samples using miRCURY RNA isolation kit - Biofluids; high-throughput bead-based protocol v.1 (Exiqon, Vedbaek, Denmark) in an automated 96-well format. The purified total RNA was eluted in a final volume of $50 \mu \mathrm{L}$. The RNA was stored in a $-80^{\circ} \mathrm{C}$ freezer.

\section{miRNA selection}

Since very little information on miRNAs in GH excess is available, the selection of miRNAs to be measured in this study was based on previously published data evaluating the relationship between miRNAs and bone phenotypes $(19,21,27,28)$. miR-99a-5p, miR-125a-5p, miR-885-5p, miR-324-5p, miR-335-3p, miR-7-1-3p, miR-22-3p, miR-660-5p and miR-10b-5p were selected based on a preliminary screening, which was performed in six patients and six controls using miRCURY LNATM Universal RT microRNA PCR Serum/Plasma Focus panel (Exiqon) (Supplementary Table 1, see section on supplementary data given at the end of this article). miR-451a and miR-23a-3p were included for hemolysis assessment of samples.

\section{MicroRNA real-time qPCR}

miRNA qPCR and further data analyses were conducted at Exiqon Services, Denmark. Using the miRCURY LNA Universal RT microRNA PCR, Polyadenylation and cDNA synthesis kit (Exiqon), $2 \mu \mathrm{L}$ RNA was reverse transcribed in $10 \mu \mathrm{L}$ reactions. Then, cDNA was diluted $50 \times$ and assayed in $10 \mu \mathrm{L}$ PCR reactions according to the protocol for miRCURY LNA Universal RT microRNA PCR; each microRNA was assayed once by qPCR on the microRNA Ready-to-Use PCR, Custom Pick and Mix panel using ExiLENT SYBR Green master mix. Negative controls excluding template from the reverse transcription reaction was performed and profiled like the samples. The amplification was performed in a LightCycler 480 Real-Time PCR System (Roche) in 384well plates. The amplification curves were analyzed using the Roche LC software, both for determination of $\mathrm{Cq}$ (by the 2nd derivative method) and for melting curve analysis.

The amplification efficiency was calculated using algorithms similar to the LinReg software. All assays were inspected for distinct melting curves and the Tm was checked to be within known specifications for the assay. Furthermore, assays must be detected with five Cqs less than the negative control, and with $\mathrm{Cq}<37$ to be included in the data analysis. Data that did not pass these criteria were omitted from any further analysis. Cq was calculated as the 2 nd derivative.

Using NormFinder the best normalizer was found to be the average of assays detected in all samples. All data were normalized to the average of assays detected in all samples (average-assay Cq). Data quality control, unsupervised

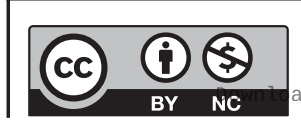

This work is licensed under a Creative Commons Attribution-NonCommercial 4.0 International License. ded from Bioscientifica.com at 04/26/2023 02:16:19PM 
data analysis and Student $t$-test with Benjamini-Hochberg correction were performed (corrected $P$ values $<0.05$ were accepted as significant).

\section{Hemolysis assessment}

MicroRNA contamination from hemolysis (29) was assessed using miR-451 (expressed in red blood cells) and the miRNA relatively stable in serum, miR-23a. The ratio between these two microRNAs correlates to degree of hemolysis. Samples with ratios above 7.0 are considered as affected by hemolysis.

\section{Statistical analysis}

The data are expressed as the mean \pm s.D., except for data that were not normally distributed, in which case median values and ranges are reported. Comparisons between two groups were performed using Student's $t$ (Gaussian distribution) and Mann-Whitney's $U$ (non-Gaussian distribution) tests, depending on the data distribution. Correlations were assessed using the Pearson's correlation coefficient or Spearman rank order depending on whether the data were normally distributed. Correlations were adjusted for age, sex, BMI and gonadal status by a stepwise multiple linear regression analysis. Tests were two-tailed, and a $P<0.05$ was considered significant.

\section{Results}

\section{Clinical characteristics and bone parameters}

The characteristics of the study population are shown in Table 1.

Volumetric bone mineral density at lumbar spine (LSvBMD), as assessed by QCT, was lower in ACRO patients as compared with healthy controls $(106 \pm 39$ vs $\left.129 \pm 30 \mathrm{mg} / \mathrm{cm}^{3} ; P=0.040\right)$. A reduction of cortical vBMD was also found in ACRO patients as compared with controls at the level of the total hip $\left(831 \pm 57\right.$ vs $883 \pm 65 \mathrm{mg} / \mathrm{cm}^{3}$; $P=0.014)$, femoral neck $\left(817 \pm 90\right.$ vs $902 \pm 159 \mathrm{mg} / \mathrm{cm}^{3}$; $P=0.037)$ and trochanter (742 (86) vs $848(204) \mathrm{mg} / \mathrm{cm}^{3}$; $P=0.006)$. CSA at the proximal femur was greater in ACRO than controls $\left(9.9 \pm 2\right.$ vs $\left.8 \pm 2 \mathrm{~cm}^{2}, P=0.031\right)$.

When postmenopausal women were ruled out from the analysis, cortical vBMD at total hip and trochanter were still reduced in ACRO patients as compared with their healthy, eugonadal counterpart (total hip,
$125 \pm 17$ vs $142 \pm 25 \mathrm{mg} / \mathrm{cm}^{3}, \quad P=0.005$; trochanter, 727 (87) vs 809 (228) $\mathrm{mg} / \mathrm{cm}^{3} ; P=0.012$ ). When only postmenopausal women were compared, no differences in bone parameters were found.

Osteoprotegerin and DKK1 levels were significantly higher in ACRO as compared with controls $(4.6 \pm 1.5$ vs $3.9 \pm 0.8 \mathrm{pmol} / \mathrm{L}, \quad P=0.047$ for osteoprotegerin; $35 \pm 15 \mathrm{pmol} / \mathrm{L}$ vs $25 \pm 14 \mathrm{pmol} / \mathrm{L}, P=0.011$ for DKK1).

\section{Differentially expressed microRNAs ACRO vs controls}

The hemolysis assessment of the samples showed no sign of red blood cell contamination. When comparing the ACRO group to the control group, the following miRNAs were found to be differentially expressed: miR-103a-3p and miR-191-5p were overexpressed in ACRO patients, whereas miR 660-5p and miR-25-3p were underexpressed in ACRO patients. miR-25-3p did not pass the BenjaminiHochberg correction (Table 2).

\section{Relationship between serum miRNAs and bone parameters}

Significant correlations between differentially expressed miRNAs and bone parameters in ACRO patients are shown in Table 3. miR-103a-3p levels were negatively associated with both trabecular vBMD at trochanter ( $r-0.38, P=0.047$ ) and serum OPG concentrations ( $r-0.41, P=0.032$ ). Levels of miR-103a-3p were also positively associated with vitamin $\mathrm{D}$ concentrations ( $r$ 0.54, $P=0.003$ ) and total CSA (Spearman's rho 0.43, $P=0.045)$. In contrast, levels of miR-660-5p were positively correlated with both trabecular vBMD at trochanter $(r 0.38, P=0.047)$ and OPG concentrations ( $r$ 0.47, $P=0.012$ ), but were negatively associated with vitamin D levels $(r-0.44, P=0.022)$. Levels of miR-25-3p were positively correlated with osteocalcin concentrations $(r 0.38, P=0.047)$.

In controls (Table 4), miR-103a-3p levels were positively associated with CTx concentrations ( $r 0.45$, $P=0.017)$ and TBS ( $r$ 0.51, $P=0.006)$, and miR-660-5p levels were negatively associated with TBS $(r-0.43$, $P=0.023)$. Levels of miR-25-3p were negatively associated with lumbar spine aBMD $(r-0.43, P=0.030)$.

No correlations were detected between miR-191-5p and bone parameters.

miR-103-a-3p was negatively associated with miR-660-5p in both ACRO patients $(r-0.86, P<0.001)$ 
Table 1 General, biochemical and bone characteristics of 27 patients with controlled acromegaly (ACRO) and 27 healthy controls.

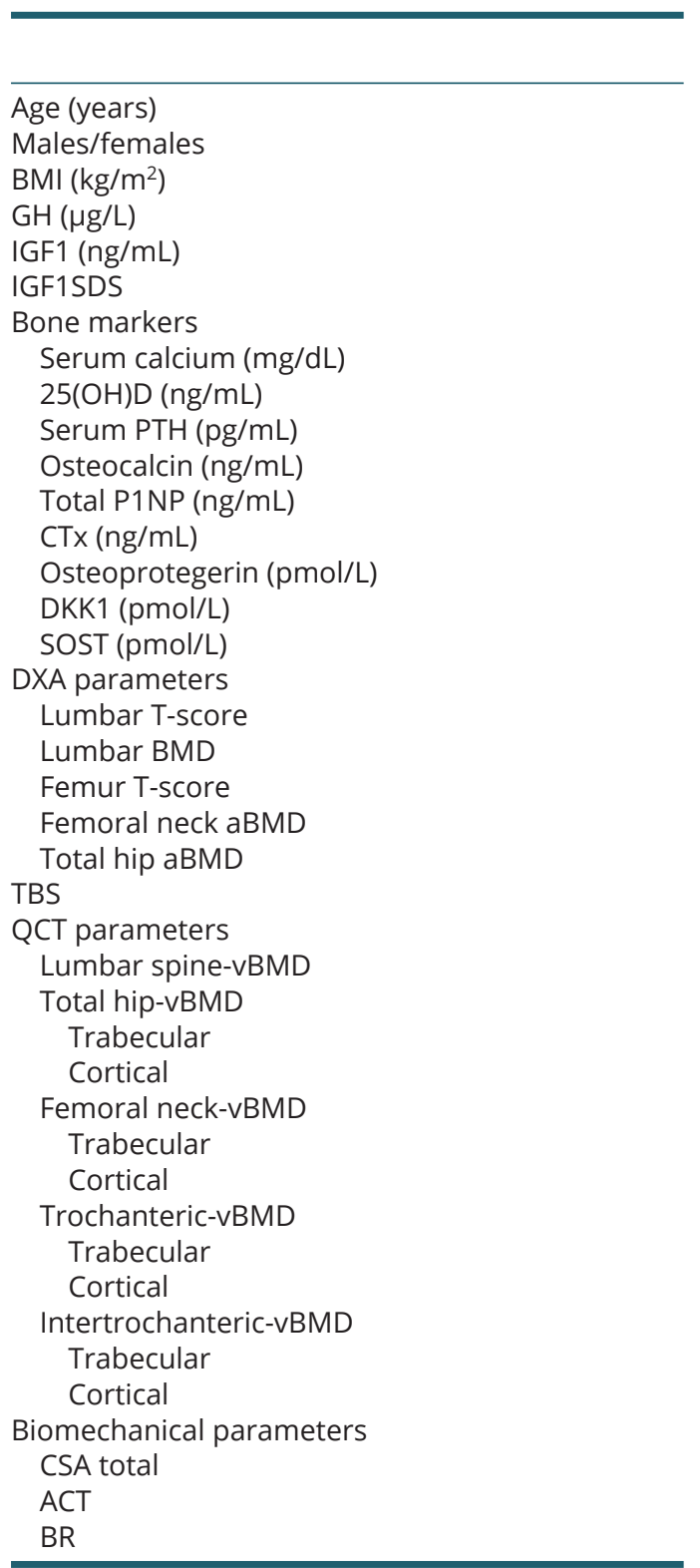

\begin{tabular}{c}
\hline ACRO $(n=27)$ \\
\hline $48 \pm 5$ \\
$11 / 16$ \\
$28 \pm 4$ \\
$1.3 \pm 1.5$ \\
$177 \pm 68$ \\
$0.9 \pm 1$ \\
$9.6 \pm 0.5$ \\
$66 \pm 27$ \\
$38 \pm 19$ \\
$17 \pm 8$ \\
$45 \pm 22$ \\
$0.35 \pm 0.18$ \\
$4.6 \pm 1.5$ \\
$35 \pm 15$ \\
$39 \pm 6$ \\
$-1.2 \pm 1.1$ \\
$0.91 \pm 0.13$ \\
$-0.6 \pm 0.98$ \\
$0.90 \pm 0.13$ \\
$0.81 \pm 0.11$ \\
$1.26 \pm 0.13$ \\
$106 \pm 39$ \\
$128 \pm 21$ \\
$831 \pm 57$ \\
$132 \pm 28$ \\
$817 \pm 90$ \\
$128 \pm 19$ \\
$742(86)$ \\
$127 \pm 25$ \\
\\
\\
\\
\\
\\
\\
\end{tabular}

\begin{tabular}{c}
\hline Controls $(n=27)$ \\
\hline $50 \pm 8$ \\
$11 / 16$ \\
$27 \pm 5$ \\
$1.4 \pm 2$ \\
$148 \pm 33$ \\
$0.3 \pm 0.7$ \\
$9 \pm 1.6$ \\
$64 \pm 35$ \\
$36 \pm 12$ \\
$19 \pm 6$ \\
$54 \pm 23$ \\
$0.39 \pm 0.18$ \\
$3.9 \pm 0.8$ \\
$25 \pm 14$ \\
$38 \pm 5$ \\
$-0.8 \pm 1.3$ \\
$0.94 \pm 0.12$ \\
$-0.36 \pm 1$ \\
$0.94 \pm 0.14$ \\
$0.81 \pm 0.12$ \\
$1.3 \pm 0.14$ \\
$129 \pm 30$ \\
$141 \pm 24$ \\
$883 \pm 65$ \\
$147 \pm 27$ \\
$902 \pm 159$ \\
$137 \pm 20$ \\
$848(204)$ \\
$143 \pm 28$ \\
$882 \pm 38$ \\
$8.4 \pm 2$ \\
\end{tabular}

\begin{tabular}{c}
\hline P value \\
\hline 0.57 \\
\\
0.87 \\
0.89 \\
0.13 \\
0.10 \\
\\
0.25 \\
0.79 \\
0.88 \\
0.42 \\
0.17 \\
0.51 \\
$\mathbf{0 . 0 4 7}$ \\
$\mathbf{0 . 0 1 1}$ \\
0.92 \\
\\
0.30 \\
0.54 \\
0.39 \\
0.39 \\
0.97 \\
0.14 \\
\end{tabular}

aStudent's $t$-test comparing patients with acromegaly vs healthy controls.

25(OH)D, 25-hydroxyvitamin D; ACT (average cortical thickness) is expressed as cm; BMI, body mass index; BR, buckling ratio is unitless; CSA, cross-sectional area is expressed as $\mathrm{cm}^{2}$; variables are expressed as mean ( \pm s.D.) or median (interquartile range) depending upon the distribution; CTX, carboxy-terminal collagen crosslinks; DKK1, Dickkopf-related protein 1; DXA, dual-energy X-ray absorptiometry; IGF-1, insulin-like growth factor-l; P1NP, type 1 amino-terminal propeptide; PTH, parathyroid hormone; QCT, quantitative computed tomography; SOST, sclerostin; TBS, trabecular bone score; vBMD, (volumetric bone mineral density) is expressed as $\mathrm{mg} / \mathrm{cm}^{3}$. Statistically significant differences $(P<0.05)$ are marked in bold.

and controls $(r-0.70, P<0.001)$. In controls only, miR-191-5p was negatively associated with miR-25-3p $(r-0.80, P<0.001)$.

No correlations were found between any of the miRNAs analyzed and the duration of either active or controlled disease.

\section{Discussion}

We have demonstrated that levels of circulating miR-103-a-3p, miR-660-5p and miR-191-5p are differentially expressed in serum of patients with controlled acromegaly (ACRO) as compared with https://ec.bioscientifica.com

https://doi.org/10.1530/EC-18-0482 (c) 2019 The authors Published by Bioscientifica Ltd

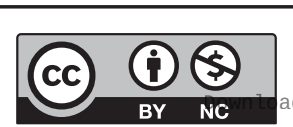

This work is licensed under a Creative Commons Attribution-NonCommercial 4.0 International License. ded from Bioscientifica.com at 04/26/2023 02:16:19PM via free access 
Table 2 miRNA expression levels in 27 controlled ACRO patients and 27 sex-, age- and BMI-matched controls.

\begin{tabular}{l}
\hline miRNA \\
\hline hsa-miR-103a-3p \\
hsa-miR-660-5p \\
hsa-miR-191-5p \\
hsa-miR-25-3p \\
hsa-miR-320a \\
hsa-miR-21-5p \\
hsa-miR-29b-3p \\
hsa-miR-125a-5p \\
hsa-miR-22-3p \\
hsa-miR-324-5p \\
hsa-miR-29a-3p \\
hsa-miR-122-5p \\
hsa-miR-335-3p \\
hsa-miR-885-5p \\
hsa-miR-382-3p \\
hsa-miR-125b-5p \\
hsa-miR-10b-5p \\
hsa-miR-7-1-3p \\
hsa-miR-99a-5p \\
hsa-miR-223-3p
\end{tabular}

\begin{tabular}{c} 
ACRO \\
\hline $1.1 \pm 0.003$ \\
$-2.8 \pm 0.005$ \\
$-0.74 \pm 0.002$ \\
$0.21 \pm 0.006$ \\
$1.02 \pm 0.004$ \\
$2.64 \pm 0.002$ \\
$-4.69 \pm 0.006$ \\
$-0.26 \pm 0.005$ \\
$-0.21 \pm 0.006$ \\
$-0.44 \pm 0.008$ \\
$-2.65 \pm 0.006$ \\
$-1.88 \pm 1.02$ \\
$-7.2 \pm 0.009$ \\
$-0.6 \pm 1.1$ \\
$-7.3 \pm 0.13$ \\
$-4.1 \pm 0.006$ \\
$-3.22 \pm 0.006$ \\
$-0.57 \pm 0.007$ \\
$-4.34 \pm 0.008$ \\
$4.81 \pm 0.004$
\end{tabular}

\begin{tabular}{c}
\hline Controls \\
\hline $0.007 \pm 0.004$ \\
$-2.2 \pm 0.07$ \\
$-0.009 \pm 0.003$ \\
$0.006 \pm 0.007$ \\
$1.3 \pm 0.007$ \\
$2.82 \pm 0.004$ \\
$-0.43 \pm 0.007$ \\
$-2.87 \pm 0.005$ \\
$-1.88 \pm 0.008$ \\
$-4.77 \pm 0.008$ \\
$-2.43 \pm 0.007$ \\
$-0.15 \pm 0.13$ \\
$-0.06 \pm 0.009$ \\
$-6 \pm 1.12$ \\
$-0.79 \pm 1.76$ \\
$-4 \pm 0.007$ \\
$-3 \pm 0.008$ \\
$-5.79 \pm 1.12$ \\
$-4.27 \pm 0.008$ \\
$-4.79 \pm 0.006$ \\
\hline
\end{tabular}

\begin{tabular}{c}
\hline Fold change \\
\hline 1.3 \\
-1.47 \\
1.17 \\
-1.32 \\
-1.21 \\
-1.13 \\
-1.24 \\
1.19 \\
-1.2 \\
1.23 \\
-1.16 \\
-0.12 \\
-0.11 \\
-1.19 \\
0.15 \\
-1.07 \\
-1.07 \\
1.06 \\
-1.05 \\
1
\end{tabular}

\begin{tabular}{c}
\hline P value \\
\hline$<0.001$ \\
$<0.01$ \\
$<0.01$ \\
0.041 \\
0.085 \\
0.088 \\
0.099 \\
0.12 \\
0.17 \\
0.17 \\
0.23 \\
0.25 \\
0.39 \\
0.41 \\
0.47 \\
0.58 \\
0.61 \\
0.74 \\
0.75 \\
0.93
\end{tabular}

\begin{tabular}{ll}
\hline Q value \\
\hline $\mathbf{0 . 0 1 3}$ \\
$\mathbf{0 . 0 2 8}$ \\
$\mathbf{0 . 0 4 4}$ \\
0.18 \\
0.27 \\
0.27 \\
0.27 \\
0.30 \\
0.35 \\
0.35 \\
0.43 \\
0.44 \\
0.60 \\
0.60 \\
0.61 \\
0.71 \\
0.71 \\
0.79 \\
0.79 \\
0.93
\end{tabular}

Bold values indicate the miRNAs that passed the false-discovery rate $(F D R)$ for multiple comparisons $(Q$ value $<0.05)$. Values shown are those with a nominal $P$ value $<0.05$ which was obtained using Student's $t$-test paired with data normalized by log 2 transformation. $Q$ values were calculated as estimates of the multiple-testing FDR. ACRO, acromegaly; miRNA, microRNA.

age-, sex- and BMI-matched controls. Moreover, we have found independent relationships between parameters of bone status and both miR-103a-3p and miR-660-5p levels that are exclusive for ACRO patients.

As a matter of fact, patients with active ACRO present with a disproportionate increase in bone turnover, especially resorption, reduced spine and femoral vBMD at both trabecular and cortical compartment, altered trabecular microstructure and elevated fracture risk at lumbar spine $(6,7,8,30)$. After remission of chronic GH/IGF-I increase, patients with controlled ACRO maintain long-lasting impairment of trabecular microarchitecture, which is associated with persistently increased risk of vertebral fractures regardless of aBMD
$(6,7,8)$. Interestingly, recent evidence suggests that the cortical compartment is also affected in ACRO patients with fragility vertebral fractures even long term after biochemical control $(7,9,31,32)$.

Mechanisms underlying the detrimental effects of GH/IGF-I excess on bone structure are multifactorial and still to be entirely elucidated (1). The interaction of GH/IGF-I with the RANK-L/OPG system may play a role in the regulation of bone metabolism. In particular, it has been suggested that IGF-I may enhance osteoclastogenesis via stimulation of the RANK-L, whereas GH may attenuate this effect by inducing OPG synthesis (33). Of note, we have found that OPG levels are greater in controlled ACRO patients compared with those in

Table 3 Correlations between miRNA expression and bone markers or bone parameters as assessed by dual-energy absorptiometry (DXA) and quantitative computed tomography (QCT) in 27 patients with acromegaly, after adjusting for age, sex, $\mathrm{BMI}$ and gonadal status.

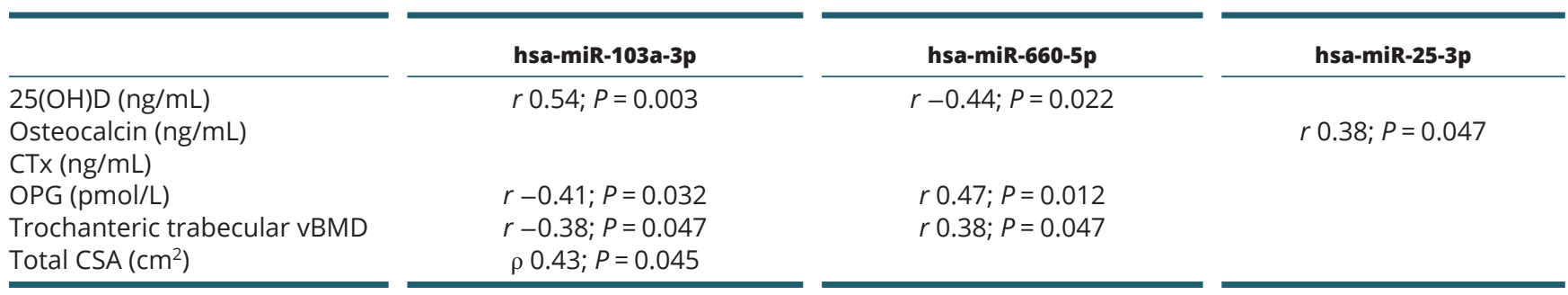

25(OH)D, 25-hydroxyvitaminD; BMD, bone mineral density; CSA, cross-sectional area; CTx, carboxy-terminal collagen crosslinks; OPG, osteoprotegerin; $\mathrm{P} 1 \mathrm{NP}$, total procollagen type 1 amino-terminal propetide; TBS, trabecular bone score; vBMD, volumetric bone mineral density. 
Table 4 Correlations between miRNA expression and bone markers or bone parameters (as assessed by DXA or QCT) in 27 healthy subjects after adjusting for sex, age, BMI and gonadal status.

\begin{tabular}{|c|c|c|c|}
\hline & hsa-miR-103a-3p & hsa-miR-660-5p & hsa-miR-25-3p \\
\hline $\mathrm{CTx}(\mathrm{ng} / \mathrm{mL})$ & $r 0.45 ; P=0.017$ & & \\
\hline Lumbar spine aBMD & & & $r-0.43 ; P=0.030$ \\
\hline TBS & $r 0.51 ; P=0.006$ & $r-0.43 ; P=0.023$ & \\
\hline
\end{tabular}

aBMD, areal bone mineral density; BMD, bone mineral density; CTx, carboxy-terminal collagen crosslinks; OPG, osteoprotegerin; SOST, sclerostin; TBS, trabecular bone score; vBMD, volumetric bone mineral density.

healthy subjects, as a possible compensatory mechanism, which counteracts increased bone resorption. Constantin et al. reported no change in the RANK-L/OPG ratio after surgical control of ACRO as compared with baseline, suggesting sustained perturbations of this system despite hormone normalization (34). Inour study, miR-103a-3p, upregulated in ACRO, was negatively associated with OPG and trabecular vBMD at the trochanter. It has been recently found that miR-103a, which is expressed in both osteoblasts and osteoclasts of postmenopausal women, negatively regulates osteoblast differentiation and bone formation under mechanical loading, by repressing the expression of RUNX2, a key transcriptional factor for osteogenesis $(27,35)$. Valenti et al. described a RUNX2 overexpression in MSCs during both the active and controlled phase of the disease, which was also associated with bone quality impairment, as assessed through histomorphometric analyses (36). As a matter of fact, both RUNX2 deletion and overexpression led to impaired bone formation $(37,38)$. However, Belaya et al. reported unchanged expression of RUNX2 in bone samples of active ACRO patients, whereas no studies have been published thus far on controlled ACRO patients (22). It could be speculated that, in controlled patients, miR-103a participates in this complex network of signals modulating both osteoclastogenesis and osteoblastogenesis, also through the regulation of the RANK-L/OPG system.

In contrast with miR-103a-3p, the miR-660-5p, downregulated in ACRO patients, positively correlated with both trabecular vBMD at trochanter and OPG. As far as we know, there is no information about the miR$660-5 p$ 's role in bone tissue, and the present study shows, for the first time, an association between this miRNA and bone.

miR-103a-3p and miR-660-5p appear to have an opposite relationship with parameters of bone metabolism, including vitamin D. While 1,25 (OH)2D levels have been described as normal or high during the active phase of the disease, its levels, as well as those of $25(\mathrm{OH}) \mathrm{D}$, did not change after control of GH/IGF-I excess $(34,39,40)$.

Our results suggest that miR-103a-3p and miR-660-5p could be potential markers of bone status and could predict the persistence of bone abnormalities in controlled ACRO patients. Noteworthy, these miRs were related to bone measurements even in healthy subjects. In particular, there was an opposite relationship between miR and TBS, while miR-103a-3p was positively associated with CTx, a marker of bone resorption. Such findings strengthen the hypothesis that miR-103a-3p and miR-660-5p play a physiological role in regulating bone homeostasis and, therefore, may represent potential signatures for bone deterioration under pathological conditions like GH/IGF-I excess.

We have also found that miR-191-5p was upregulated in our patients. However, we did not detect any relationships between its levels and any of the bone parameters we measured. We are not aware of any putative role of this miRNAs in bone regulation and, therefore, we hypothesize that this finding may be independent of skeletal health.

As we previously described, DKK1 levels were higher in ACRO patients as compared with controls, but no relationship was found between this Wnt signaling inhibitor and any of the miRNAs analyzed (41).

Limitations of our study include small sample size and relative heterogeneity of our population. In particular, $19 \%$ of patients were in pharmacological remission and $26 \%$ received radiotherapy. It is currently not known whether pharmacological treatment of ACRO may cause independent skeletal actions on bone or interfere with miRNA expression (42). Nevertheless, none of the miRNAs described here have been reported as differentially expressed in GH-secreting pituitary adenomas from patients who are responders vs nonresponders to somatostatin analogs (28). Ten of our patients had also undergone radiotherapy previously, but Biermasz et al. demonstrated that previous pituitary irradiation was not associated with alteration of lumbar spine BMD in ACRO 
patients in remission (43). Similarly, we could not assess the impact of comorbidities on miRNA expression in our patients. Another limitation of this study is its crosssectional design, which prevents from inferring causality from our data. Lack of information on vertebral fractures as well as lack of a group with active acromegaly are the other limitations of this study.

Since the source of the miRNAs in serum is currently unknown, we cannot exclude that alterations of miRNA expression may reflect specific actions in other tissues. miRNAs are ubiquitous modulators controlling multiple patterns of gene expression and, therefore, may play a pathogenic role in several tissues and/or may be markers of more than one ACRO-related complication.

Indeed, ACRO is a multisystemic disease, which also seems to be associated with an elevated risk of malignancies (44). It is intriguing to speculate that miR103a-3p, miR-660-5p and miR-191-5p, which have been related to oncogenesis both in vitro and in vivo, may also be involved in the occurrence of tumors in ACRO patients $(45,46,47,48,49,50)$.

Furthermore, associations of miRNAs and bone parameters remained significant after adjusting for the gonadal status, suggesting that previous exposure to $\mathrm{GH} /$ IGF-I excess is the main determinant of bone impairment in patients with controlled ACRO, thus counteracting the negative effect of concomitant hypogonadism. Indeed, Madeira et al. found deteriorated trabecular microarchitecture at both the distal radius and distal tibia, as assessed by high-resolution peripheral QCT (HR-pQCT), in eugonadal patients with controlled ACRO, as compared with their hypogonadal counterpart (6). Similarly, Godang et al. showed that TBS was reduced in ACRO eugonadal men after 1 year of hormone excess correction, but not in hypogonadal subjects (39). Moreover, another study reported no associations between the gonadal status of controlled ACRO patients and trabecular vBMD measurements across the proximal femur and also showed that controlled ACRO with normal gonadal function had lower vBMD at both total hip and intertrochanter as compared with controls (8).

In conclusion, we have described that some miRNAs are differentially expressed in the serum of ACRO patients as compared with controls and are associated with both biochemical and structural parameters of bone metabolism. In particular, our data, which need to be confirmed in larger studies, suggest that miR-103a-3p and miR-660-5p may be promising biomarkers to evaluate the presence of bone disease in GH/IGF-I excess and assess the persistence of bone impairment even after biochemical control has been reached.

\section{Supplementary data}

This is linked to the online version of the paper at https://doi.org/10.1530/ EC-18-0482.

\section{Declaration of interest}

The authors declare that there is no conflict of interest that could be perceived as prejudicing the impartiality of the research reported.

\section{Funding}

This work was supported by grants from the Instituto de Salud Carlos III (FIS PI14/00194 and PI17/00749), FEDER funds.

\section{Acknowledgement}

A special thanks to all the patients and controls who accepted to participate in this research. The authors are indebted to Ana Maria Marín and Eulalia Urgell for their technical assistance.

\section{References}

1 Giustina A, Mazziotti G \& Canalis E. Growth hormone, insulin-like growth factors, and the skeleton. Endocrine Reviews 200829 535-559. (https://doi.org/10.1210/er.2007-0036)

2 Olarescu NC, Berryman DE, Householder LA, Lubbers ER, List EO, Benencia F, Kopchick JJ \& Bollerslev J. GH action influences adipogenesis of mouse adipose tissue-derived mesenchymal stem cells. Journal of Endocrinology 2015226 13-23. (https://doi. org/10.1530/JOE-15-0012)

3 Ueland T, Olarescu NC, Jorgensen AP, Otterdal K, Aukrust P, Godang $\mathrm{K}$, Levka T \& Bollerslev J. Increased serum and bone matrix levels of the secreted Wnt antagonist DKK-1 in patients with growth hormone deficiency in response to growth hormone treatment. Journal of Clinical Endocrinology and Metabolism 2015100 736-743. (https://doi. org/10.1210/jc.2014-2912)

4 Colao A, Ferone D, Marzullo P \& Lombardi G. Systemic complications of acromegaly: epidemiology, pathogenesis and management. Endocrine Reviews 200425 102-152. (https://doi. org/10.1210/er.2002-0022)

5 Dekkers OM, Biermasz NR, Pereira AM, Romijn JA \& Vandenbroucke JP. Mortality in acromegaly: a metaanalysis. Journal of Clinical Endocrinology and Metabolism 200893 61-67. (https://doi. org/10.1210/jc.2007-1191)

6 Madeira M, Neto LV, de Paula Paranhos Neto F, Barbosa Lima IC, Carvalho de Mendonça LM, Gadelha MR \& Fleiuss de Farias ML. Acromegaly has a negative influence on trabecular bone, but not on cortical bone, as assessed by high-resolution peripheral quantitative computed tomography. Journal of Clinical Endocrinology and Metabolism 201398 1734-1741. (https://doi.org/10.1210/jc.20124073)

7 Maffezzoni F, Maddalo M, Frara S, Mezzone M, Zorza I, Baruffaldi F, Doglietto F, Mazziotti G \& Maroldi R. High-resolution beam tomography analysis of bone microarchitecture in patients with acromegaly and radiological vertebral fractures. Endocrine 201654 532-542. (https://doi.org/10.1007/s12020-016-1078-3)

8 Valassi E, Crespo I, Malouf J, Llauger J, Aulinas A, Marin AM, Biagetti B \& Webb SM. Reduction of trabecular and cortical volumetric bone
This work is licensed under a Creative Commons Attribution-NonCommercial 4.0 International License. ded from Bioscientifica.com at 04/26/2023 02:16:19PM 
mineral density at the proximal femur in patients with acromegaly. European Journal of Endocrinology 2016174 107-114. (https://doi. org/10.1530/EJE-15-0931)

9 Malgo F, Hamdy NAT, Rebelink TJ, Kroon HM, Claessen KMJA, Pereira AM, Biermasz NR \& Appelman-Dijkstra NM. Bone material strength index as measured by impact micriindentation is altered in patients with acromegaly. European Journal of Endocrinology 2017176 339-347. (https://doi.org/10.1530/EJE-16-0808)

10 Kocijan R, Muschitz C, Geiger E, Skalicky S, Baierl A, Dormann R, Plachel F, Feichtinger X, Heimel P, Fahrleitner-Pammer A, et al. Circulating microRNA signatures in patients with idiopathic and postmenopausal osteoporosis and fagility fractures. Journal of Clinical Endocrinology and Metabolism 2016101 4125-4134. (https://doi. org/10.1210/jc.2016-2365)

11 Hassan MQ, Tye CE, Stein GS \& Lian JB. Non-coding RNAs : epigenetic regulators of bone development and homeostasis. Bone 201581 746-756. (https://doi.org/10.1016/j.bone.2015.05.026)

12 Turchinovich A, Weiz L \& Burwinker B. Extracellular miRNAs: the mistery of their origin and function. Trends in Biochemical Sciences 201237 460-465. (https://doi.org/10.1016/j.tibs.2012.08.003)

13 Gordon JAR, Montecino MA, Aqueilan RI, Stein JL, Stein GS \& Lian JB. Epigenetic pathways regulating bone homeostasis: potential targeting for intervention of skeletal disorders. Current Osteoporosis Reports 201412 496-506. (https://doi.org/10.1007/s11914-0140240-1)

14 Cao Z, Moore BT, Wang Y, Peng XH, Lappe JM, Recker RR \& Xiao P. MiR-422a as a potential cellular microRNA biomarker for postmenopausal osteoporosis. PLoS ONE 20149 e97098. (https://doi. org/10.1371/journal.pone.0097098)

15 Li H, Wang Z, Fu Q \& Zhang J. Plasma miRNA levels correlate with sensitivity to bone mineral density in postmenopausal osteoporosis patients. Biomarkers 201419 553-556. (https://doi.org/10.3109/1354 750X.2014.935957)

16 Bedene A, Mencej Bedrač S, Ješe L, Marc J, Vrtačnik P, Preželj J, Kocjan T, Kranjc T, Ostanek B MiR-148a the epigenetic regulator of bone homeostasis is increased in plasma of osteoporotic postmenopausal women. Wiener klinische Wochenschrift 2016128 (Supplement 16) 519-526. (https://doi.org/10.1007/s00508-0161141-3)

17 Seeliger C, Karpinski K, Haug, Vester H, Schmitt A, Bauer JS \& van Griensven M Five freely circulating miRNAs and bone tissue miRNAs are associated with osteoporotic fractures. Journal of Bone and Mineral Research 201419 553-556. (https://doi.org/10.1002/jbmr.2175)

18 Weilner S, Skalicky S, Salzer B, Keider V, Wagner F, Hildner F, Gabriel C, Dovjak P, Pietschmann P, Grillari-Voglauer R, et al. Differentially circulating miRNAs after recent osteoporotic fracture can influence osteogenic differentiation. Bone 201579 43-51. (https://doi. org/10.1016/j.bone.2015.05.027)

19 Panach L, Mifsut D, Tarín JJ, Cano A \& Garcia-Pérez MA. Serum circulating microRNAs as biomarkers of osteoporotic fracture. Calcified Tissue International 201597 495-505. (https://doi. org/10.1007/s00223-015-0036-z)

20 Heilmeier U, Hackl M, Skalicky S, Weilner S, Schroeder F, Vierlinger K, Patsch JM, Baum T, Oberbauer E, Lobach I, et al. Serum miRNA Signatures are indicative of skeletal fractures in postmenopausal women with and without type 2 diabetes and influence osteogenic and adipogenic differentiation of adipose tissue-derived mesenchymal stem cells in vitro. Journal of Bone and Mineral Research 201631 2173-2192. (https://doi.org/10.1002/jbmr.2897)

21 Yavropoulou MP, Anastasilakis A, Makras P, Tsalikakis D, Grammatiki $\mathrm{M} \&$ Yovos JG. Expression of microRNAs that regulate bone turnover in the serum of postmenopausal women with low bone mass and vertebral fractures. European Journal of Endocrinology $2017 \mathbf{1 7 6}$ 169-176. (https://doi.org/10.1530/EJE-16-0583)

22 Belaya Z, Grebennikova T, Melnichenko GA, Nikitin A, Solodovnikov A, Brovkina A, Grigoriev A, Rozhinskaya L, Lutsenko A
\& Dedov LL. Effects of active acromegaly on bone mRNA and microRNA expression patterns. European Journal of Endocrinology 2018 178 353-364. (https://doi.org/10.1530/EJE-17-0772)

23 Aulinas A, Crespo I, Vilades D, Leta R, Urgell E, Biagetti B, Webb SM \& Valassi E. Cystatin-C and epicardial adipose tissue as noninvasive predictors of cardiovascular risk in acromegaly. Clinical Endocrinology 201686 214-222. (https://doi.org/10.1111/cen.13273)

24 Giustina A, Chanson P, Bronstein MD, Klibanski A, Lamberts S, Casanueva FF, Trainer P, Ghigo E, Ho K \& Melmed S. A consensus on criteria for cure of acromegaly. Journal of Clinical Endocrinology and Metabolism 201095 3141-3148. (https://doi.org/10.1210/jc.20092670)

25 Molitch ME, Clemmons DR, Malozowski S, Merriam GR \& Vance ML. Evaluation and treatment of adult growth hormone deficiency: an Endocrine Society Clinical Practice Guidelines. Journal of Clinical Endocrinology and Metabolism 201196 1587-1609. (https://doi. org/10.1210/jc.2011-0179)

26 Allison SJ, Poole KES, Treece GM, Gee AH, Tonkin C, Rennie WJ, Folland JP, Summers GD \& Brooke-Wavell K. The influence of high impact exercise on cortical and trabecular bone mineral content and 3D distribution across the proximal femur in older men: a randomised controlled unilateral intervention. Journal of Bone and Mineral Research 201530

1709-1716. (https://doi.org/10.1002/jbmr.2499)

27 Zuo B, Zhu J, Li J, Wang C, Zhao X, Cai G, Li Z, Peng J, Wang P \& Shen C, et al. microRNA-103a functions as a mechanosensitive microRNA to inhibit bone formation through targeting Runx2. Journal of Bone and Mineral Research 201430 330-345. (https://doi. org/10.1002/jbmr.2352)

28 Mao ZG, Sheng He D, Zhou J, Yao B, Xiao W, Chen CH, Zhu YH \& Wang JJ. Differential expression of microRNAs in GH secreting pituitary adenomas. Diagnostic Pathology 20105 79. (https://doi. org/10.1186/1746-1596-5-79)

29 Blondal T, Jensby Nielsen S, Baker A, Andreasen D, Mouritzen P, Wrang Teilum M \& Dahlsveen IK. Assessing sample and miRNA profile quality in serum and plasma or other biofluids. Methods 201359 S1-S6. (https://doi.org/10.1016/j. ymeth.2012.09.015)

30 Mazziotti G, Frara S \& Giustina A. Pituitary diseases and bone. Endocrine Reviews 201839 440-488. (https://doi.org/10.1210/er.201800005)

31 Dalle Carbonare L, Micheletti V, Cosaro E, Valenti MT, Mottes M, Francia G \& Daví MV. Bone histomorphometry in acromegaly patients with fragility vertebral fractures. Pituitary 201821 56-64. (https://doi.org/10.1007/s11102-017-0847-1)

32 Mazziotti G, Biagioli E, Maffezzoni F, Spinello M, Serra V, Maroldi R, Floriani I \& Giustina A. Bone turnover, bone mineral density, and fracture risk in acromegaly: a meta-analysis. Journal of Clinical Endocrinology and Metabolism 2015100 384-394. (https://doi. org/10.1210/jc.2014-2937)

33 Lanzi R, Losa M, Villa I, Gatti E, Sirtori M, Dal Fiume C \& Rubinacci A. GH replacement therapy increases plasma osteoprotegerin levels in GH-deficient adults. European Journal of Endocrinology 2003148 185-191. (https://doi.org/10.1530/eje.0.1480185)

34 Constantin T, Tangpricha V, Shah R, Oyesiku NM, Ioachimescu OC, Ritchie J \& Ioachimescu AG. Calcium and bone turnover markers in acromegaly: a prospective, controlled study. Journal of Clinical Endocrinology and Metabolism 2017102 2416-2424 (https://doi. org/10.1210/jc.2016-3693)

35 De-Ugarte L, Serra-Vinardell J, Nonell L, Belcells S, Arnal M, Nogues M, Mellibovski L, Grinberg D, Diez-Perez A \& García-Giralt N. Expression profiling of microRNAs in human bone tissue from postmenopausal women. Human Cell 201831 33-41. (https://doi. org/10.1007/s13577-017-0181-y)

36 Valenti MT, Mottes, M, Cheri S, Deiana M, Micheletti V, Corsaro E \& Daví MV, Francia G \& Dalle Carbonare L. Runx2 overexpression

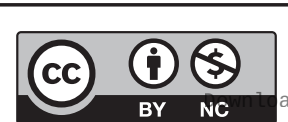

This work is licensed under a Creative Commons Attribution-NonCommercial 4.0 International License. ded from Bioscientifica com at 04/26/2023 02:16:19PM 
compromises bonr quality in acromegaly. Endocrine-Related Cancer 201825 269-277. (https://doi.org/10.1530/ERC-17-0523)

37 Komori T, Yagi H, Nomura S, Yamaguchi A, Sasaki K, Deguchi K, Shimizu Y, Bronson RT, Gao YH, Inada M, et al. Targeted disruption of Cbfa1 results in a complete lack of bone formation owing to maturational arrest of osteoblasts. Cell 199789 755-765. (https://doi. org/10.1016/S0092-8674(00)80258-5)

38 Cohen MM Jr. Perspectives on RUNX genes: an update. American Journal of Medical Genetics Part A 2009149 2629-2646. (https://doi. org/10.1002/ajmg.a.33021)

39 Godang K, Olarescu NC, Bollerslev J \& Heck A. Treatment of acromegaly increases BMD but reduced trabecular bone score: a longitudinal study. European Journal of Endocrinology 2016175 155-164. (https://doi.org/10.1530/EJE-16-0340)

40 Shah R, Licata A, Oyesiku NM \& Ioachimescu AG. Acromegaly as a cause of 1,15-dihydroxyvitamin D-dependent hypercalcemia: case reports and review of the literature. Pituitary 201215 S17-S22. (https://doi.org/10.1007/s11102-010-0286-8)

41 Valassi E, Crespo I, Malouf J, Vilades D, Leta R, Llauger J, Urgell E, Aulinas A, Marin AM, Biagetti B, et al. Epicardial fat is a negative predictor of spine volumetric bone mineral density and trabecular bone score in acromegaly. Endocrine 201653 860-864. (https://doi. org/10.1007/s12020-016-0945-2)

42 Giustina A, Chanson P, Kleinberg D, Bronstein MD, Clemmons DR, Klibanski A, van der Lely AJ, Strasburger CJ, Lamberts SW, Ho KKY, et al. Expert consensus document: a consensus on the medical treatment of acromegaly. Nature Reviews Endocrinology 201410 243-248. (https://doi.org/10.1038/ nrendo.2014.21)

43 Biermasz NR, Hamdy NAT, Pereira AM, Romijn JA \& Roelfesma F. Long-term maintenance of the anabolic effects of GH on the skeleton in successfully treated patients with acromegaly. European Journal of Endocrinology 2005152 53-60. (https://doi.org/10.1530/ eje.1.01820)
44 Melmed S, Colao A, Barkan A, Molitch M, Grossman AB, Kleinberg D, Clemmons D, Chanson P, Laws E, Schlechte J, et al. Guideline for acromegaly management. An update. Journal of Clinical Endocrinology and Metabolism 200994 1509-1517. (https://doi.org/10.1210/ jc.2008-2421)

45 Zhang JX, Song W, Chen ZH, Wei JH, Liao YJ, Lei J, Hu M, Chen GZ, Liao B, Lu J, et al. Prognostic and predictive value of a microRNA signature in stage II colon cancer: a microRNA expression analysis. Lancet Oncology 201314 1295-1236. (https://doi.org/10.1016/S14702045(13)70491-1)

46 Fasihi A, Soltani BM, Atashi A \& Nasiri S. Introduction of hsa-miR103a and hsa-miR-1827 and hsa-miR-137 as new regulators of Wnt signaling pathway and their relation to colorectal carcinoma. Journal of Cell Biochemistry $2018 \mathbf{1 1 9}$ 5104-5117. (https://doi.org/10.1002/jcb.26357)

47 Shen Y, Ye YF, Ruan LW, Bao L, Wu MW \& Zhou Y. Inhibition of miR660-5p expression suppresses tumor development and metastasis in human breast cancer. Genetics and Molecular Research 20172316. (https://doi.org/10.4238/gmr16019479)

48 Bye A, Røsiø H, Nauman J, Silva GJ, Follestad T, Omland T \& Wisløff U. Circulating microRNAs predict future fatal myocardial infarction in healthy individuals - The HUNT study. Journal of Molecular and Cellular Cardiology 201697 162-168. (https://doi.org/10.1016/j. yjmcc.2016.05.009)

49 Jakob P, Kacprowski T, Briand-Schumacher S, Heg D, Klingenberg R, Stähli BE, Jaguszewski M, Rodondi N, Nanchen D, Räber L, et al. Profiling and validation of circulating microRNAs for cardiovascular events in patients presenting with ST-segment elevation myocardial infarction. European Heart Journal 201738 511-515. (https://doi. org/10.1093/eurheartj/ehw563)

50 Chen P, Pan X, Zhao L, Jin L, Lin C, Quan J, He T, Zhou L, Wu X, Wang Y, et al. MicroRNA-191-5p exerts a tumor suppressive role in renal cell carcinoma. Experimental and Therapeutic Medicine 201815 1686-1693. (https://doi.org/10.3892/etm.2017.5581)

Received in final form 18 December 2018

Accepted 21 December 2018

Accepted Preprint published online 21 December 2018
This work is licensed under a Creative Commons Attribution-NonCommercial 4.0 International License. ded from Bioscientifica.com at $04 / 26 / 2023$ 02:16:19PM 